\title{
Chronic Stress Contributes to Cognitive Dysfunction and Hippocampal Metabolic Abnormalities in APP/PS1 Mice
}

\author{
Bing Han ${ }^{a}$ Jin-Hua Wang ${ }^{a}$ Yuan Geng ${ }^{b}$ Li Shenc Hua-Long Wanga \\ Yan-Yong Wang ${ }^{a}$ Ming-Wei Wanga,b \\ aDepartment of Neurology, The First Hospital of Hebei Medical University, Shijiazhuang, \\ bBrain Aging and Cognitive Neuroscience Laboratory of Hebei province, Shijiazhuang, \\ 'Clinical laboratory, The First Hospital of Hebei Medical University, Shijiazhuang, Hebei, PR China
}

\section{Key Words}

Alzheimer's disease $\bullet$ Stress response $\bullet$ Transgenic mice $•$ Metabolomics $•$ Gas chromatographymass spectrometry

\begin{abstract}
Background/Aims: Stress response is determined by the brain, and the brain is a sensitive target for stress. Our previous experiments have confirmed that once the stress response is beyond the tolerable limit of the brain, particularly that of the hippocampus, it will have deleterious effects on hippocampal structure and function; however, the metabolic mechanisms for this are not well understood. Methods: Here, we used morris water maze, elisa and gas chromatography-time of flight/mass spectrometry to observe the changes in cognition, neuropathology and metabolomics in the hippocampus of APP/PS1 mice and wildtype (C57) mice caused by chronic unpredictable mild stress (CUMS), we also further explored the correlation between cognition and metabolomics. Results: We found that 4 weeks of CUMS aggravated cognitive impairment and increased amyloid- $\beta$ deposition in APP/PS1 mice, but did not affect C57 mice. Under non-stress conditions, compared with C57 mice, there were 8 different metabolites in APP/PS1 mice. However, following CUMS, 3 different metabolites were changed compared with untreated C57 mice. Compared to APP/PS1 mice, there were 7 different metabolites in APP/PS1+CUMS mice. Among these alterations, 3-hydroxybutyric acid, valine, serine, beta-alanine and o-phosphorylethanolamine, which are involved in sphingolipid metabolism, synthesis and degradation of ketone bodies, and amino acid metabolism. Conclusion: The results indicate that APP/PS1 mice are more vulnerable to stress than C57 mice, and the metabolic mechanisms of stress-related cognitive impairment in APP/ PS1 mice are related to multiple pathways and networks, including sphingolipid metabolism, synthesis and degradation of ketone bodies, and amino acid metabolism.
\end{abstract}




\section{Cellular Physiology Cell Physiol Biochem 2017;41:1766-1776 \\ \begin{tabular}{l|l} 
and Biochemistry Publisher 10.1159/000471869 & $\begin{array}{l}\text { (c) } 2017 \text { The Author(s). Published by S. Karger AG, Basel } \\
\text { www.karger.com/cpb }\end{array}$
\end{tabular} \\ Han et al.: Chronic Stress Aggravates Cognition-Related Metabolic Abnormalities in APP/ PS1 Mice}

\section{Introduction}

Stress is a nonspecific and adaptive response caused by social environmental factors. Even when subjected to similar stressors, there are individual difference in the stress response, because some people are more vulnerable to stress and may subsequently experience a change in homeostasis and disease, while others can better tolerate similar stressful experiences $[1,2]$. An efficient stress tolerance not only requires the rapid activation of the HPA axis, but also the effective termination of that activation when the stressor disappears or remains present for a long time $[3,4]$. Both important processes depend on the modulation of the brain by the nervous, endocrine and immune systems $[5,6]$. In addition, the brain is a sensitive target organ for stress; if the stress intensity exceeds tolerable limits, it will result in the development of mental and somatic disease [7, 8]. Many experiments have established that chronic stress is a risk factor for the development of cognitive deficits, especially for Alzheimer's disease (AD). Chronic stress not only impaired the early longterm potentiation (LTP) in the at-risk rat model of $\mathrm{AD}$ [9], but it also decreased hippocampal synaptic plasticity [10] and increased amyloid- $\beta$ plaque deposition in Tg2576 mice [11]. Furthermore, our previous study also confirmed that chronic stress exposure increased the levels of glucocorticoids (1.5-fold), promoted senile plaque deposition, neuronal injury, and cognitive impairment in APP/PS1 mice compared to wild-type mice [12]; however, the cognition-related metabolic mechanisms during chronic stress are not well understood.

Metabolomics is the systematic study of small-molecule metabolites in a biological cell, tissue or organ, and it offers an opportunity to better understand the outcomes of some biological processes $[13,14]$. The metabolite biomarkers discovered by metabolic profiling analyses help in determining the diagnosis and prognosis of clinical diseases and also help to comprehensively characterize phenotypes, which are influenced by both genetic and environmental factors [15]. Among the metabolomics technology platforms, gas chromatography-time of flight/mass spectrometry (GC-TOF/MS) has been demonstrated to be an efficient tool for the reliable detection and accurate mass measurement of metabolites $[16,17]$.

Chronic unpredictable mild stress (CUMS) is recognized to be a robust model of the stress response, consisting of a schedule of mild psychosocial and physiological stressors that last for several weeks. Recently, many studies have reported metabolic perturbations of the brain [18], urine [19] and plasma [20] associated with the application of the CUMS model. As a classic model of AD, APP/PS1 double transgenic mice express both the human APPswe and PS1-dE9 mutations, develop elevated levels of A 342 at 3-4 months, and present with cognitive deficits at 6 months, which progress with age [21,22]. Moreover, neuropathological and neurochemical studies have shown that aging, disease, and the presence of gene mutations are all risk factors for stress overload $[11,23]$. The purpose of our study was to investigate the relationship between chronic stress and $\mathrm{AD}$, and to further explore whether the presence of AD-associated genetic mutations (APP/PS1) affect the metabolic profile of the hippocampus during chronic stress.

\section{Materials and Methods}

\section{Animals}

Breeding pairs of B6.C3-Tg (APPswe, PSEN1dE9)85Dbo/NJU (for short, APP/PS1) and C57BL/6Nju (for short, C57) were purchased from Nanjing Bio medical Research Institute. All of the 6-month-old APP/ PS1 and C57 male mice were randomly divided into four groups (C57, n=11; C57+CUMS, n=12; APP/PS1, $\mathrm{n}=12$; and APP/PS1+CUMS, $\mathrm{n}=10$ ). Non-CUMS mice were housed in groups of 2-3 under a 12-hour lightdark cycle. Food and water were provided ad libitum. CUMS mice were housed individually in another room, with food, water, and light-dark cycle conditions according to the CUMS procedure. The temperature of rooms was maintained at $22^{\circ} \mathrm{C} \pm 2{ }^{\circ} \mathrm{C}$. All animal experiments and protocol were approved by the ethics committee of Hebei Medical University.

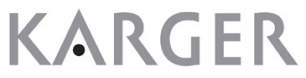




\section{Cellular Physiology Cell Physiol Biochem 2017;41:1766-1776

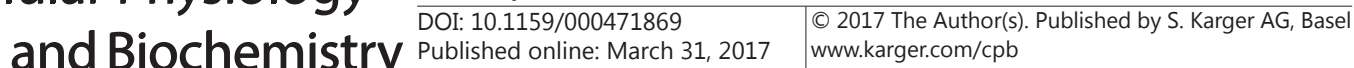

Han et al.: Chronic Stress Aggravates Cognition-Related Metabolic Abnormalities in APP/ PS1 Mice

\section{CUMS}

The CUMS procedure used in the present was previously validated in mice [24], with some modifications. Mice were exposed to social and environmental stressors 2-3 times a day for 4 weeks. The stressors included 1) food deprivation for $24 \mathrm{~h}, 2$ ) water deprivation for $24 \mathrm{~h}$ with an empty bottle for the last $1 \mathrm{~h}, 3$ ) overnight illumination, 4) removal sawdust for $24 \mathrm{~h}, 5$ ) soiled cage (200 ml of water in $100 \mathrm{~g}$ of sawdust bedding) for $24 \mathrm{~h}, 6)$ forced swimming at $8{ }^{\circ} \mathrm{C}$ for $\left.6 \mathrm{~min}, 7\right)$ tail nipping $(1 \mathrm{~cm}$ from the tip of the tail), and 8) physical restraint for $2 \mathrm{~h}$. Different stressors were applied in a pseudo-random manner each day, with the sequence of stressors changed weekly. The detailed process was shown in our previously paper [10].

\section{Morris water maze}

MWM was tested on the day following 3 weeks of CUMS. The maze consisted of a black circular tank (120-cm diameter) filled with water $\left(20-22^{\circ} \mathrm{C}\right)$ that was made opaque with the addition of a nontoxic white pigment to obscure the platform. The tank was divided into 4 imaginary quadrants, with a transparent platform submerged $1 \mathrm{~cm}$ below the water surface in the middle of one quadrant. During the place navigation test, mice were released once at each of the four start positions on each day in 5 consecutive days. Mice were given $60 \mathrm{~s}$ to find the platform during each trial, and the time taken to escape (escape latency) was measured. If a mouse failed to locate the platform within $60 \mathrm{~s}$, it was guided onto the platform, where it stayed for $10 \mathrm{~s}$, and it was recorded as $60 \mathrm{~s}$. After $24 \mathrm{~h}$, in the probe trial, the platform was removed and the mice was allowed to swim for $60 \mathrm{~s}$ in search of the platform. The time spent in the target quadrant and the number of platform crossings were measured to show the platform location retention. All performance was recorded by ANY-maze Behavior Analysis System (Stoelting Co., Wood Dale, IL, USA). Data were expressed as the mean \pm SEM. Differences in the place navigation test were detected using repeated measures ANOVA with SPSS13.0. Other data were analyzed by one-way ANOVA, and the statistical significances between C57 vs C57+CUMS, C57 vs APP/PS1 and APP/PS1 vs APP/PS1+CUMS were evaluated by the least significant difference (LSD) post-hoc analysis, with $p<0.05$ considered statistically significant.

\section{A $\beta$ Elisa}

Mice were sacrificed by decapitation after behavioral test. Hippocampus was quickly separated, weighed by microbalance, stored at $-80{ }^{\circ} \mathrm{C}$ and homogenized in 5 volumes of guanidine-Tris buffer $(5 \mathrm{M}$ guanidine $\mathrm{HCl} / 50 \mathrm{mM}$ Tris- $\mathrm{HCl}, \mathrm{pH}$ 8.0). Mix the homogenates at room temperature for $4 \mathrm{~h}$ and centrifuge with 16,000 rpm for $20 \mathrm{~min}$ at $4{ }^{\circ} \mathrm{C}$. Total protein concentration was quantified using a BCA Protein Assay (Solarbio, Beijing, China). Levels of $A \beta 40$ and $A \beta 42$ were detected using a high sensitivity ELISA kit (Novex, Life Technologies, USA) according to the manufacturer's instructions. Read each sample's absorbance at $450 \mathrm{~nm}$.

\section{Tissue sample pretreatment}

For GC-TOF/MS analysis, the CA1 region of hippocampus was isolated, weighed and extracted with 10 volumes of methanol-chloroform (3: 1, v/v) in Eppendorf tubes [25]. The mixture was homogenized at $65 \mathrm{~Hz}$ for $3 \mathrm{~min}$ and centrifuged at $4^{\circ} \mathrm{C}$ at 12,000 rpm for $15 \mathrm{~min}$. Eighty microlitres of supernatant was transferred into a 2-ml GC-MS glass vial. The supernatant was dried in a vacuum concentrator at $37^{\circ} \mathrm{C}$ for $3 \mathrm{~h}, 20 \mu \mathrm{l}$ of methoxylamine hydrochloride (dissolved in pyridine, final concentration of $20 \mathrm{mg} / \mathrm{ml}$ ) was added, and the sample was incubated at $80^{\circ} \mathrm{C}$ for $20 \mathrm{~min}$ in an oven after mixing and sealing. An additional $30 \mu \mathrm{l}$ of BSTFA (containing 1\% TCMS, v/v) was quickly added to each sample, and the sample was incubated at $70^{\circ} \mathrm{C}$ for $1 \mathrm{~h}$. After cooling, FAMEs ( $5 \mu \mathrm{l}$, standard mixture of fatty acid methyl esters, C8-C16:1 mg/ml; $\mathrm{C} 18-\mathrm{C} 24: 0.5 \mathrm{mg} / \mathrm{ml}$ in chloroform) was added to the sample for calculating retention index (RI, an indicator of qualitative analysis). The mixture sample was subjected to detection by GC-TOF/MS.

\section{GC-TOF/MS analysis}

GC-TOF/MS analysis was performed using an Agilent 7890 gas chromatograph system coupled with a Pegasus HT time-of-flight mass spectrometer. The system utilized an Rxi-5Sil MS column $(30 \mathrm{~m} \times 250 \mu \mathrm{m}$ inner diameter, $0.25 \mu \mathrm{m}$ film thickness; Restek, USA). Helium was used as the carrier gas, with a front inlet purge flow of $3 \mathrm{ml} / \mathrm{min}$ and a gas flow rate through the column of $1 \mathrm{ml} / \mathrm{min}$. The initial temperature was kept at $50^{\circ} \mathrm{C}$ for $1 \mathrm{~min}$, raised to $330^{\circ} \mathrm{C}$ at a rate of $10^{\circ} \mathrm{C} / \mathrm{min}$, and then maintained at $330^{\circ} \mathrm{C}$ for $5 \mathrm{~min}$. The 
injection, transfer line, and ion source temperatures were $280^{\circ} \mathrm{C}, 280^{\circ} \mathrm{C}$, and $250^{\circ} \mathrm{C}$, respectively. The energy was $-70 \mathrm{eV}$ in electron impact mode. The mass spectrometry data were acquired in full-scan mode with a $\mathrm{m} / \mathrm{z}$ range of 85 to 600 at a rate of 20 spectra/sec after a solvent delay of $366 \mathrm{~s}$.

\section{GC-TOF/MS Data processing}

Chroma TOF4.3X software of LECO Corporation and the LECO-Fiehn Rtx5 database were used for raw peaks exaction, data baselines filtration and calibration, peak alignment, deconvolution analysis, peak identification and integration of the peak area [26]. The RI was used in the peak identification, and the RI tolerance was 5000. For the GC-Quad FiehnLib library, the derivatives by increasing numbers were according to RI, e.g., serine 1, serine 2 and serine 3 (for the derivatives with no, one or two trimethylsilylgroups derivatizing the primary amino group.). Noise was removed by the interquartile range method, and missing values of raw data were imputed by half of the minimum value; 567 peaks and 487 metabolites were detected. In addition, the peak area normalization method was used in this data analysis. The resulting threedimensional data, including the peak number, sample name, and normalized peak area, were entered into the SIMCA13 software package (Umetrics, Umea, Sweden) for principal component analysis (PCA), partial least squares-discriminant analysis (PLS-DA) and orthogonal projections to latent structures-discriminate analysis (OPLS-DA). PCA was used to display natural separation among groups by visual inspection of score plots, as well as identify clustering trend. PLS-DA was validated to check the interpretation (R2Y) and the prediction (Q2Y) of model. To obtain a higher level of group separation and a better understanding of variables responsible for classification, potential candidates were chosen based on the contribution of Variable Importance for the Projection (VIP) extracted from the first principal component of the OPLS-DA analysis. VIP>1.0 was first selected, and the remaining variables were then assessed by Student's T test, with $p<0.05$ considered statistically significant between two comparison groups.

\section{Metabolite identification and correlation analysis}

In addition, the LECO/Fiehn Metabolomics Library was used to evaluate the identification of the discriminating compound. A similarity $>700$ indicated that the accuracy of the metabolite identification was reliable. The correlation analysis was conducted between the time spent in target quadrant and the metabolite identification, the Pearson correlation coefficient $|r|>0.5$ and $p<0.05$ were selected as cognitionrelated metabolites during stress. The selected metabolites were further validated and cross listed with the metabolism pathway by Kyoto Encyclopedia of Genes and Genomes (KEGG), and finally, a sketch was constructed according to the potential functional analysis.

\section{Results}

\section{Effects of stress on cognitive function}

In the place navigation test, the differences in escape latency among the groups showed statistical significance $(F=7.924, p<0.001)$. On the fifth day, the escape latency was increased in APP/PS1 mice (vs C57 mice, $t=-2.534, p=0.015$ ) and further extended in APP/PS1+CUMS ( $v s$ APP/PS1 mice, $t=-2.220, p=0.032$ ); whereas, there was no statistically significant difference in escape latency between C57 and C57+CUMS mice (Fig. 1A, B). In the probe trial, there was a significant difference $(F=9.407, p<0.001)$ in the time spent in target quadrant, LSD post-hoc tests demonstrated that it was significantly decreased in APP / PS1 mice ( $v s$ C57 mice, $t=2.427, p=0.020$ ), and further reduced in APP/PS1+CUMS mice ( $v s$ APP/PS1 mice, $t=2.185, p=0.035$ ) (Fig. 1C). No significant differences were shown in the number of platform crossings in C57+CUMS mice and APP/PS1 mice compared with that of C57 mice; furthermore, it was significantly lower in APP/PS1+CUMS mice than in APP/PS1 mice $(t=2.193, p=0.034$, Fig. 1D).

Effects of stress on A levels

$\mathrm{A} \beta 40$ and $\mathrm{A} \beta 42$ ELISA results showed a significant effect among groups $(F=6.787$, $p=0.004$; and $F=8.464, p=0.001$ ). Post-hoc tests showed that the levels of $A \beta 40$ and $A \beta 42$ were increased in APP/PS1 mice ( $v s$ C57 mice, $t=-2.592, p=0.020$; and $t=-2.104, p=0.051$ ) 




Fig. 1. CUMS-induced cognitive deficiency. (A, B) The escape latency of MWM was increased in APP/PS1 mice compared with C57 mice and in APP/PS1+CUMS compared with APP/PS1 mice. (C) The time spent in the target quadrant was decreased in APP/PS1 mice compared with C57 mice and in APP/PS1+CUMS compared with APP/PS1 mice. (D) The number of platform crossings in APP/PS1+CUMS mice was significantly fewer than in APP/PS1 mice. ${ }^{*} p<0.05$ vs C57 group, $\# p<0.05$ vs APP/PS1 group, $\mathrm{n}=10$-12 per group.

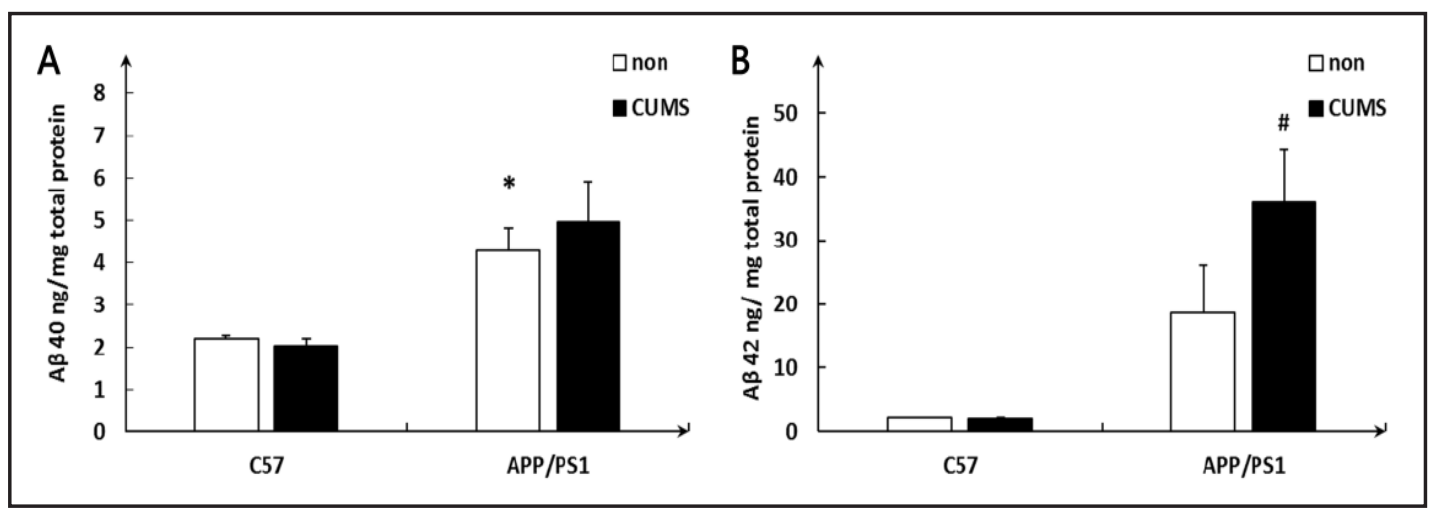

Fig. 2. Effects of stress on $A \beta$ levels. (A) The levels of A $\beta 40$ were increased in APP/PS1 mice compared with C57 mice. (B) The levels of A 342 were increased in APP/PS1+CUMS mice compared with APP/PS1 mice. ${ }^{*} p<0.05$ vs C57 group, $\# p<0.05$ vs APP/PS1 group, $\mathrm{n}=5$ per group.

and that CUMS further upregulated the levels of A 342 in APP/PS1+CUMS mice (vs APP/PS1 mice, $t=-2.190, p=0.044)$; however no effect of CUMS on A $\beta 40$ and A $\beta 42$ levels was detected in C57 mice and C57+CUMS mice (Fig. 2).

\section{Effects of stress on the metabolic Profile}

Multivariate analyses were performed using SIMCA-P 13.0. An unsupervised PCA test showed a clear separation between each group that was paired for analysis, except for the C57 and the C57+CUMS groups (Fig. 3 A, B, C). As a supervised clustering analysis, PLSDA model was applied to understand the variables for classification. The R2Y values of 

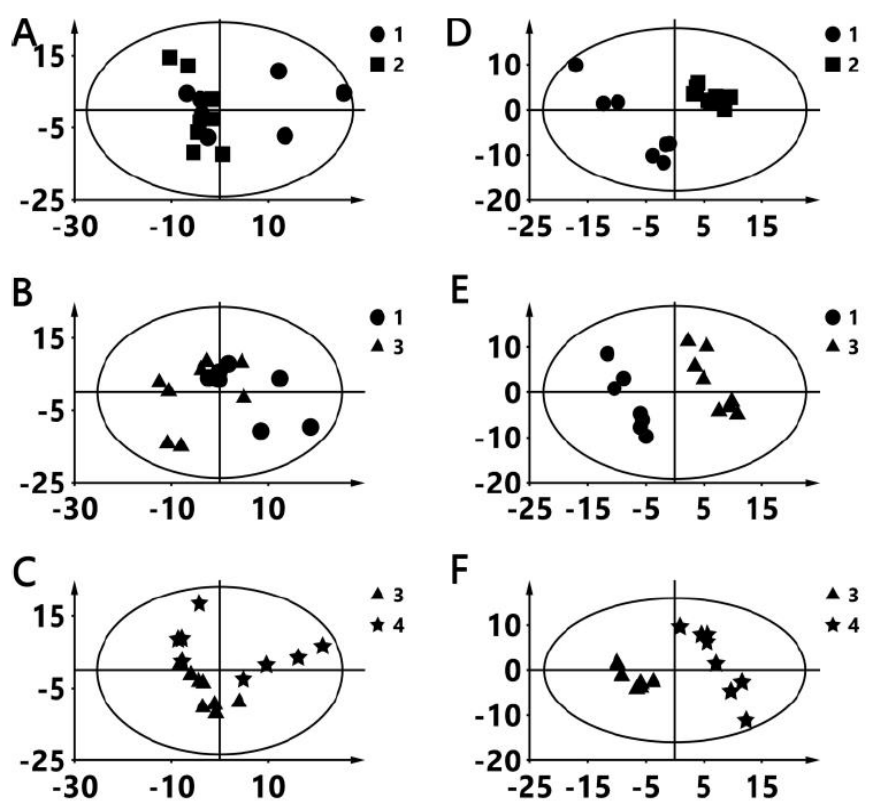

E

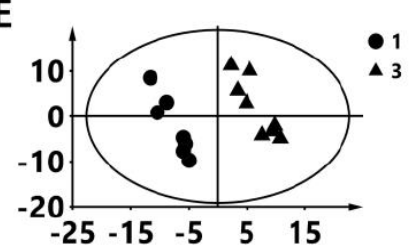

$\mathrm{F}$

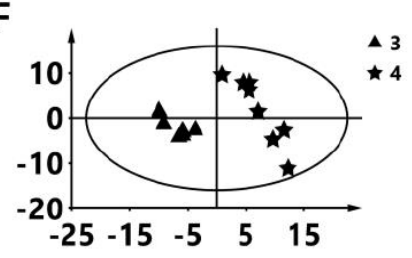

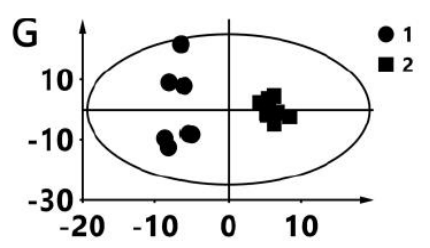

$\mathrm{H}$

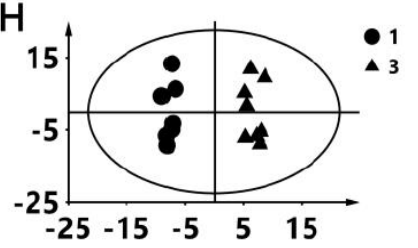

I

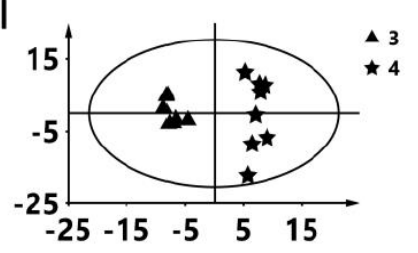

Fig. 3. PCA score plots (A, B, C), PLS-DA (D, E, F) and OPLS-DA (G, H, I) analyses with all of the metabolites. PLS-DA and OPLS-DA showed that the samples from different groups were scattered into different regions. C57 group ( $\bullet, n=7)$; C57+CUMS group $(\boldsymbol{\bullet}, \mathrm{n}=8)$; APP/PS1 group $(\boldsymbol{\Lambda}, \mathrm{n}=8)$; APP/PS1+CUMS group $(\star, n=8)$. Each sample represents one mouse.

Table 1. Metabolite changes were observed in APP/PS1 and C57 mice during CUMS. R.I. represents the retention index of the peak; $\uparrow$ or $\downarrow$ represents the changing trend of metabolites when the former is compared with the latter; $\Delta$ represents variable importance for the projection (VIP) $>1$; *represents $p<0.05$ vs C57; \#represents $p<0.05$ vs APP/PS1

\begin{tabular}{lccccc}
\hline & Similarity & R.I. & C57+CUMS & APP/PS1 & APP/PS1+CUMS vs APP/PS1 \\
& & & vs C57 & vs C57 & \\
\hline 3-hydroxybutyric acid & 868.77419 & $8.6146,0$ & $\uparrow$ & $\uparrow$ & $\Delta \uparrow \#$ \\
valine & 716.61538 & $9.38278,0$ & $\downarrow$ & $\Delta \downarrow^{*}$ & $\downarrow$ \\
serine 2 & 945.18519 & $10.0227,0$ & $\uparrow$ & $\downarrow$ & $\Delta \downarrow \#$ \\
threonine 2 & 937.125 & $10.5057,0$ & $\uparrow$ & $\downarrow$ & $\Delta \downarrow \#$ \\
serine 1 & 824.13043 & $11.3298,0$ & $\downarrow$ & $\downarrow$ & $\Delta \downarrow \#$ \\
beta-alanine 2 & 790.02174 & $12.2318,0$ & $\downarrow$ & $\Delta \downarrow *$ & $\downarrow$ \\
L-malic acid & 938.69118 & $12.9754,0$ & $\uparrow$ & $\downarrow$ & $\Delta \uparrow \#$ \\
o-phosphorylethanolamine & 925.77358 & $16.3614,0$ & $\downarrow$ & $\Delta \downarrow *$ & $\Delta \downarrow \#$ \\
sorbose 2 & 734.88406 & $17.3458,0$ & $\uparrow$ & $\Delta \uparrow^{*}$ & $\downarrow$ \\
pantothenic acid & 795.77612 & $18.477,0$ & $\downarrow$ & $\Delta \downarrow^{*}$ & $\Delta \uparrow \#$ \\
palmitic acid & 919.68421 & $19.0469,0$ & $\Delta \uparrow^{*}$ & $\Delta \uparrow^{*}$ & $\downarrow$ \\
stearic acid & 882.18841 & $20.8242,0$ & $\Delta \uparrow^{*}$ & $\Delta \uparrow^{*}$ & $\downarrow$ \\
1-monopalmitin & 913.69565 & $23.5411,0$ & $\Delta \downarrow *$ & $\downarrow$ & $\uparrow$ \\
monostearin & 859.55072 & $24.9436,0$ & $\downarrow$ & $\uparrow$ & $\Delta \downarrow \#$ \\
\hline
\end{tabular}

C57 vs C57+CUMS, C57 vs APP/PS1 and APP/PS1 vs APP/PS1+CUMS were 0.993, 0.998 and 0.993 , respectively, and the Q2Y values were $0.574,0.692$ and 0.68 , respectively; taken together, these results suggested that the model was stable and functioned as an accurate predictor (Fig. 3 D, E, F). Furthermore, to refine the PLS-DA analysis, an OPLS-DA analysis was performed to maximize the differences between groups; all samples fell inside the $95 \%$ 
Table 2. The cognition-related metabolic abnormalities after CUMS. Pearson correlation analysis was conducted between the time spent in target quadrant and the metabolite identification, the correlation coefficient $|r|>0.5$ and $p<0.05$ were selected as cognition-related metabolites during stress. + indicates positive correlations; - indicates negative correlations

\begin{tabular}{lccccc}
\hline & 3-hydroxybutyric acid & valine & serine & beta-alanine & o-phosphorylethanolamine \\
\hline Coefficient & -0.705 & +0.502 & +0.571 & +0.527 & +0.822 \\
$p$ & $<0.001$ & 0.004 & 0.001 & 0.002 & $<0.001$ \\
\hline
\end{tabular}

Hotelling T2 ellipse (Fig. 3 G, H, I).

Under the non-stress condition, significant differences were found between C57 mice and APP/PS1 mice in the levels of 8 metabolites. The levels of valine, beta-alanine, o-phosphorylethanolamine and pantothenic acid were significantly decreased in APP/ PS1 mice; the levels of sorbose, palmitic acid and stearic acid were increased in APP/PS1 mice. Following CUMS, relative to C57 mice, 1-monopalmitin levels were decreased, and the palmitic acid and stearic acid levels were increased in C57+CUMS mice. Compared with APP/PS1 mice, the levels of serine, threonine, o-phosphorylethanolamine and monostearin were significantly decreased; in contrast, the levels of 3-hydroxybutyric acid, L-malic acid and pantothenic acid were increased in APP/PS1+CUMS mice (Table 1).

\section{Correlation between cognition and metabolic profiles}

Pearson correlation analyses were conducted between the time spent in the target quadrant and the presence of each metabolite. As presented in Table 2, the cognitionrelated metabolites affected by CUMS were 3-hydroxybutyric acid $(r=-0.705, p<0.001)$, valine $(r=+0.502, p=0.004)$, serine $(r=+0.571, p=0.001)$, beta-alanine $(r=+0.527, p=0.002)$ and $o$-phosphorylethanolamine $(r=+0.822, p<0.001)$. By using online KEGG, the cognitionrelated metabolites involved in sphingolipid metabolism, synthesis and degradation of ketone bodies, and amino acid metabolism.

\section{Discussion}

Consistent with our previous study, we found that APP/PS1 mice showed impaired memory, increased A $\beta$ deposition in hippocampus, and CUMS exposure further aggravated the cognitive impairments and neuropathology at 6 months APP/PS1 mice. While the difference from previous study was no significant impact on the time spent in target quadrant after suffer from CUMS. We believe that, on the one hand, it's might be related to the fact that 6-month-old C57 mice have longer tolerance time of stress and they are at a peak developmental age for optimal brain function; on the other hand, it's connected with the body function condition of mice, the techniques of experimenter, the animal's habits in different seasons, and so on. Well, taken together all these experiments indicated that the stress tolerance was different between C57 and APP/PS1 mice, to further elucidate the mechanisms behind these phenomena, we used GC-TOF/MS and multivariate statistics to profile cognition-related metabolites present in the brain of APP/PS1 mice and wild-type mice during exposure to stress.

Based on the normalized peak areas of the target analytes from the hippocampus, the score plot of the PLS-DA and OPLS-DA analyses showed a clear separation between groups, suggesting that CUMS exposure affected APP/PS1 and wild-type mice differently. As it contains some of the highest glucocorticoid receptor levels in the brain, the hippocampus is particularly sensitive to stress $[27,28]$. Recently, we found that although the basal levels of corticosterone were similar between C57 and APP/PS1 mice, the stress tolerance of APP/ PS1 mice was lower than age-matched C57 mice, as evidenced by dramatically increased corticosterone levels and reduced glucocorticoid receptor (GR) expression in hippocampus 
following exposure to stress [12]. Similarly, this result showed that CUMS exposure obviously disturbed the metabolic pattern of hippocampal neurons in APP/PS1 mice, while no significant effects were observed in C57 mice, further illustrating an impaired tolerance to stress in APP/PS1 mice. Additionally, the correlation analysis showed that 5 potential biomarkers (3-hydroxybutyric acid, valine, serine, beta-alanine and o-phosphorylethanolamine) were closely associated with cognitive impairment induced by CUMS.

The baseline levels of many metabolites were differences between C57 mice and APP/ PS1 mice. By GC-TOF/MS analysis, phosphorylethanolamine was obviously decreased in APP/PS1 mice compared with C57 mice, implying a defect in lipid metabolism in AD brains [29-31]. As the second largest component of brain phospholipids and a precursor of phosphatidylethanolamine (PE), low-level phosphorylethanolamine usually reflects decreased myelination [32,33]. Hypomyelination not only negatively affects the saltatory conduction of action potentials but also impairs cognitive and behavioral performance [34]. In addition, oxidative stress is another vital factor in $\mathrm{AD}$; the high metabolic rate of the brain in AD results in hypersensitivity to reactive oxygen species (ROS). PEs and/ or PE-derived unsaturated fatty acids serve as the substrate of sphingolipid peroxidation; decreases usually reflect weak antioxidant defense mechanisms of the brain, which are accompanied by increased protein oxidation, protein crosslinking and the formation of amyloid plaques and nerve fiber tangles [35, 36]. Valine, a branched-chain amino acid, can quickly offer amino groups for the synthesis of glutamate, thus maintaining brain nitrogen homeostasis and indirectly affecting excitatory neurotransmitters [37]. Beta-alanine is not only a potential neurotransmitter at glycine receptors but also a constituent of carnosine and an inhibitor of taurine transport; these biological actions suggest that the level of betaalanine in the hippocampus is probably involved in spatial memory retrieval [38]. Although no clear conclusion can be drawn $[18,29,39]$, our results were consistent with recent studies that have demonstrated that the levels of valine and total alanine were decreased in either the serum or the cerebral gray matter of AD patients [40-42]. The lower levels of these metabolites provided some new evidence regarding the confusing role of lipids and amino acids in AD.

There is evidence that stress or excessive glucocorticoids, once beyond the tolerable limit, have deleterious effects on brain structure and function; more severe damage may occur in some vulnerable groups, e.g., AD patients[11, 12, 43]. However, few studies have focused on the metabolic pattern in AD during chronic stress. In APP/PS1 mice following CUMS, the phosphorylethanolamine levels were further down-regulated, and in response to this, the $A \beta$ deposition were also significantly increased, suggesting the lipid metabolism changes caused by stress exactly accelerated the progression of neuropathological damage in AD. It is noteworthy that the 3-hydroxybutyric acid levels were obviously increased in APP/ PS1+CUMS mice; it also known as $\beta$-hydroxybutyric acid, a component of ketone bodies, and is produced from the $\beta$-oxidation of fatty acids before transfer to other tissues for use. Our previous study found that the effect of insulin resistance was enhanced in APP/PS1+CUMS mice, suggesting a dysfunction of glucose metabolism and bioenergetics in the brain; therefore, the notion that ketone body metabolism may serve as a compensatory pathway for deficits in bioenergetics was suggested, which has been confirmed in sleep-deprivation, stress, and starvation $[44,45]$. Nevertheless, research investigating the utilization of ketones is limited in AD; one study indicated a modest increase in SCOT expression (catabolizes ketone bodies into Acetyl-CoA and generates ATP), while the expression of HADHA and SCHAD (enzymes involved in fatty acid oxidation and ketogenesis) was significantly increased $3 \mathrm{x}$ in TgAD brain [46]. Although many studies reported the neuroprotective effect of ketogenic diets [47], the continued accumulation of 3-hydroxybutyric acid from non-dietary sources could diminish the interfacial viscosity of membrane lipids and modulate ion channels and thus disturb higher brain function $[48,49]$. Furthermore, our findings shown that cognitive impairment was associated with serine level aberrations after chronic stress. Unfortunately, we were unable to distinguish between L-serine and D-serine. In vivo, L-serine is produced by the serine racemase compound $\mathrm{D}$-serine. $\mathrm{D}$-serine is the main endogenous co-agonist

\section{KARGER}




\section{Cellular Physiology Cell Physiol Biochem 2017;41:1766-1776

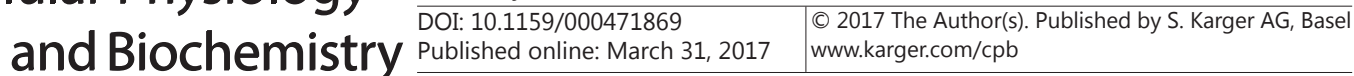 \\ Han et al.: Chronic Stress Aggravates Cognition-Related Metabolic Abnormalities in APP/ \\ PS1 Mice}

of NMDAR and is required for the expression of the synaptic plasticity involved in memory [50]. Overall, the metabolic responses affecting cognition of APP/PS1 mice following CUMS included systematic changes in sphingolipid metabolism, synthesis and degradation of ketone bodies, and amino acid metabolism. Further mechanisms remain to be explored.

In contrast to C57 mice, the cognition, neuropathology and the cognition-related metabolic patterns of APP/PS1 mice were significantly changed after exposure to CUMS. These altered metabolic patterns suggest decreased resistance to stress in APP/PS1 mice, implying that $\mathrm{AD}$-associated genetic mutations are high risk factors for lower stress tolerance. Among the metabolic alterations, 3-hydroxybutyric acid, valine, serine, beta-alanine and o-phosphorylethanolamine were considered as the potential candidate biomarkers, and they appeared to take part in the stress-related cognitive impairment in AD.

\section{Conclusion}

In conclusion, our study indicated that compared to age-matched C57 mice, social environmental factors (e.g., CUMS) aggravated cognitive dysfunction and A $\beta$ deposition, as well as perturbed hippocampal metabolism in APP/PS1 mice. The cognition-related metabolic abnormalities caused by stress exposure were related to multiple pathways and networks, including sphingolipid metabolism, synthesis and degradation of ketone bodies, and amino acid metabolism. This work warns us that, for some vulnerable groups (e.g., people carrying AD-associated genetic mutations or people who have developed AD), stress management and hormone homeostasis are of utmost importance to maintain optimal neurological function.

\section{Abbreviations}

AD (Alzheimer's disease); GC-TOF/MS (gas chromatography-time of flight/mass spectrometry); CUMS (chronic unpredictable mild stress); MWM (morris water maze); PCA (principal component analysis); PLS-DA (partial least squares-discriminant analysis); OPLS-DA (orthogonal projections to latent structures-discriminate analysis); VIP (Variable Importance for the Projection).

\section{Acknowledgments}

This work was supported by Natural Science Foundation of Hebei Province (H2015206238).

\section{Disclosure Statement}

The authors declare no conflict of interest.

\section{Reference}

1 Oitzl MS, Champagne DL, van der Veen R, de Kloet ER: Brain development under stress: hypotheses of glucocorticoid actions revisited. Neurosci Biobehav Rev 2010;34:853-866.

2 Hogan C: Chronic stress--an approach to management in general practice. Aust Fam Physician 2013;42:542-545.

3 ter Heegde F, De Rijk RH, Vinkers CH: The brain mineralocorticoid receptor and stress resilience. Psychoneuroendocrinology 2015;52:92-110.

4 McEwen BS: Protection and damage from acute and chronic stress: allostasis and allostatic overload and relevance to the pathophysiology of psychiatric disorders. Ann N Y Acad Sci 2004;1032:1-7. 


\section{Cellular Physiology Cell Physiol Biochem 2017;41:1766-1776 \begin{tabular}{c|c|c|c|} 
DOI: 10.1159/000471869 & C 2017 The Author(s). Published by S. Karger AG, Basel \\
wwww.kargercom/cpb
\end{tabular} and Biochemistry \\ Han et al.: Chronic Stress Aggravates Cognition-Related Metabolic Abnormalities in APP/}

5 Detka J, Kurek A, Basta-Kaim A, Kubera M, Lason W, Budziszewska B: Neuroendocrine link between stress, depression and diabetes. Pharmacol Rep 2013;65:1591-1600.

6 Keller-Wood M: Hypothalamic-Pituitary--Adrenal Axis-Feedback Control. Compr Physiol 2015;5:11611182.

7 Juster RP, McEwen BS, Lupien SJ: Allostatic load biomarkers of chronic stress and impact on health and cognition. Neurosci Biobehav Rev 2010;35:2-16.

8 Gulbins A, Grassme H, Hoehn R, Wilker B, Soddemann M, Kohnen M, Edwards MJ, Kornhuber J, Gulbins E: Regulation of Neuronal Stem Cell Proliferation in the Hippocampus by Endothelial Ceramide. Cell Physiol Biochem 2016;39:790-801.

-9 Alkadhi KA, Tran TT: Chronic psychosocial stress impairs early LTP but not late LTP in the dentate gyrus of at-risk rat model of Alzheimer's disease. Brain Res 2014;1588:150-158.

10 Wang J, Yuan J, Pang J, Ma J, Han B, Geng Y, Shen L, Wang H, Ma Q, Wang Y, Wang M: Effects of Chronic Stress on Cognition in Male SAMP8 Mice. Cell Physiol Biochem 2016;39:1078-1086.

11 Joshi YB, Chu J, Pratico D: Stress hormone leads to memory deficits and altered tau phosphorylation in a model of Alzheimer's disease. J Alzheimers Dis 2012;31:167-176.

12 Han B, Yu L, Geng Y, Shen L, Wang H, Wang Y, Wang J, Wang M: Chronic Stress Aggravates Cognitive Impairment and Suppresses Insulin Associated Signaling Pathway in APP/PS1 Mice. J Alzheimers Dis 2016;53:1539-1552.

13 Wang H, Geng Y, Han B, Qiang J, Li X, Sun M, Wang Q, Wang M: Repetitive transcranial magnetic stimulation applications normalized prefrontal dysfunctions and cognitive-related metabolic profiling in aged mice. PLoS One 2013;8:e81482.

-14 Koek MM, Jellema RH, van der Greef J, Tas AC, Hankemeier T: Quantitative metabolomics based on gas chromatography mass spectrometry: status and perspectives. Metabolomics 2010;7:307-328.

-15 Lindon JC, Holmes E, Nicholson JK: Metabonomics and its role in drug development and disease diagnosis. Expert Rev Mol Diagn 2004;4:189-199.

16 Lindon JC, Holmes E, Nicholson JK: Metabonomics techniques and applications to pharmaceutical research \& development. Pharm Res 2006;23:1075-1088.

-17 Čajka T, Hajšlová J: Gas chromatography-high-resolution time-of-flight mass spectrometry in pesticide residue analysis: advantages and limitations. J Chromatogr A 2004;1058:251-261.

18 Ni Y, Su M, Lin J, Wang X, Qiu Y, Zhao A, Chen T, Jia W: Metabolic profiling reveals disorder of amino acid metabolism in four brain regions from a rat model of chronic unpredictable mild stress. FEBS Lett 2008;582:2627-2636.

19 Liu XJ, Li ZY, Li ZF, Gao XX, Zhou YZ, Sun HF, Zhang LZ, Guo XQ, Du GH, Qin XM: Urinary metabonomic study using a CUMS rat model of depression. Magn Reson Chem 2012;50:187-192.

20 Gao X, Guo B, Yang L, Liu J, Zhang X, Qin X, Du G: Selection and dynamic metabolic response of rat biomarkers by metabonomics and multivariate statistical analysis combined with GC-MS. Pharmacol Biochem Behav 2014;117:85-91.

-21 Webster SJ, Bachstetter AD, Nelson PT, Schmitt FA, Van Eldik LJ: Using mice to model Alzheimer's dementia: an overview of the clinical disease and the preclinical behavioral changes in 10 mouse models. Front Genet 2014;5:88.

-22 Trinchese F, Liu S, Battaglia F, Walter S, Mathews PM, Arancio O: Progressive age-related development of Alzheimer-like pathology in APP/PS1 mice. Ann Neurol 2004;55:801-814.

-23 Solas M, Aisa B, Tordera RM, Mugueta MC, Ramirez MJ: Stress contributes to the development of central insulin resistance during aging: implications for Alzheimer's disease. Biochim Biophys Acta 2013;1832:2332-2339.

24 Liu Y, Yang N, Hao W, Zhao Q Ying T, Liu S, Li Q Liang Y, Wang T, Dong Y, Ji C, Zuo P: Dynamic proteomic analysis of protein expression profiles in whole brain of Balb/C mice subjected to unpredictable chronic mild stress: implications for depressive disorders and future therapies. Neurochem Int 2011;58:904-913.

25 Geier FM, Want EJ, Leroi AM, Bundy JG: Cross-platform comparison of Caenorhabditis elegans tissue extraction strategies for comprehensive metabolome coverage. Anal Chem 2011;83:3730-3736.

26 Kind T, Wohlgemuth G, Lee do Y, Lu Y, Palazoglu M, Shahbaz S, Fiehn O: FiehnLib: mass spectral and retention index libraries for metabolomics based on quadrupole and time-of-flight gas chromatography/ mass spectrometry. Anal Chem 2009;81:10038-10048. 


\section{Cellular Physiology Cell Physiol Biochem 2017;41:1766-1776 \begin{tabular}{l|l|l} 
and Biochemistry 10.1159/000471869 & $\begin{array}{l}\text { C) } 2017 \text { The Author(s). Published by S. Karger AG, Basel } \\
\text { www.karger.com/cpb }\end{array}$ \\
\hline
\end{tabular}}

Han et al.: Chronic Stress Aggravates Cognition-Related Metabolic Abnormalities in APP/ PS1 Mice

27 Conrad CD: Chronic stress-induced hippocampal vulnerability: the glucocorticoid vulnerability hypothesis. Rev Neurosci 2008;19:395-411.

28 Miller DB, O'Callaghan JP: Aging, stress and the hippocampus. Ageing Res Rev 2005;4:123-140.

29 Gonzalez-Dominguez R, Garcia-Barrera T, Vitorica J, Gomez-Ariza JL: Deciphering metabolic abnormalities associated with Alzheimer's disease in the APP/PS1 mouse model using integrated metabolomic approaches. Biochimie 2015;110:119-128.

-30 Nitsch RM, Blusztajn JK, Pittas AG, Slack BE, Growdon JH, Wurtman RJ: Evidence for a membrane defect in Alzheimer disease brain. Proc Natl Acad Sci USA 1992;89:1671-1675.

-31 Wang H, Lian K, Han B, Wang Y, Kuo SH, Geng Y, Qiang J, Sun M, Wang M: Age-related alterations in the metabolic profile in the hippocampus of the senescence-accelerated mouse prone 8: a spontaneous Alzheimer's disease mouse model. J Alzheimers Dis 2014;39:841-848.

- 32 Duarte JM, Lei H, Mlynarik V, Gruetter R: The neurochemical profile quantified by in vivo 1H NMR spectroscopy. Neuroimage 2012;61:342-362.

33 Pettegrew JW, Panchalingam K, Withers G, McKeag D, Strychor S: Changes in brain energy and phospholipid metabolism during development and aging in the Fischer 344 rat. J Neuropathol Exp Neurol 1990;49:237249.

- 34 Miller DJ, Duka T, Stimpson CD, Schapiro SJ, Baze WB, McArthur MJ, Fobbs AJ, Sousa AM, Sestan N, Wildman DE, Lipovich L, Kuzawa CW, Hof PR, Sherwood CC: Prolonged myelination in human neocortical evolution. Proc Natl Acad Sci USA 2012;109:16480-16485.

-35 Prasad MR, Lovell MA, Yatin M, Dhillon H, Markesbery WR: Regional membrane phospholipid alterations in Alzheimer's disease. Neurochem Res 1998;23:81-88.

-36 Gonzalez-Dominguez R, Garcia-Barrera T, Gomez-Ariza JL: Combination of metabolomic and phospholipidprofiling approaches for the study of Alzheimer's disease. J Proteomics 2014;104:37-47.

-37 Shimomura Y, Harris RA: Metabolism and physiological function of branched-chain amino acids: discussion of session 1. J Nutr 2006;136:232S-233S.

38 Sase A, Dahanayaka S, Hoger H, Wu G, Lubec G: Changes of hippocampal beta-alanine and citrulline levels are paralleling early and late phase of retrieval in the Morris Water Maze. Behav Brain Res 2013;249:104108.

-39 Bhattacharya TK, Pence BD, Ossyra JM, Gibbons TE, Perez S, McCusker RH, Kelley KW, Johnson RW, Woods JA, Rhodes JS: Exercise but not (-)-epigallocatechin-3-gallate or beta-alanine enhances physical fitness, brain plasticity, and behavioral performance in mice. Physiol Behav 2015;145:29-37.

-40 Gonzalez-Dominguez R, Garcia-Barrera T, Gomez-Ariza JL: Using direct infusion mass spectrometry for serum metabolomics in Alzheimer's disease. Anal Bioanal Chem 2014;406:7137-7148.

41 Gonzalez-Dominguez R, Garcia-Barrera T, Gomez-Ariza JL: Metabolite profiling for the identification of altered metabolic pathways in Alzheimer's disease. J Pharm Biomed Anal 2015;107:75-81.

42 D'Aniello A, Vetere A, Fisher GH, Cusano G, Chavez M, Petrucelli L: Presence of D-alanine in proteins of normal and Alzheimer human brain. Brain Res 1992;592:44-48.

43 Dong H, Goico B, Martin M, Csernansky CA, Bertchume A, Csernansky JG: Modulation of hippocampal cell proliferation, memory, and amyloid plaque deposition in APPsw (Tg2576) mutant mice by isolation stress. Neuroscience 2004;127:601-609.

44 Teague CR, Dhabhar FS, Barton RH, Beckwith-Hall B, Powell J, Cobain M, Singer B, McEwen BS, Lindon JC, Nicholson JK, Holmes E: Metabonomic studies on the physiological effects of acute and chronic psychological stress in Sprague-Dawley rats. J Proteome Res 2007;6:2080-2093.

45 Chikahisa S, Shimizu N, Shiuchi T, Sei H: Ketone body metabolism and sleep homeostasis in mice. Neuropharmacology 2014;79:399-404.

46 Yao J, Hamilton RT, Cadenas E, Brinton RD: Decline in mitochondrial bioenergetics and shift to ketogenic profile in brain during reproductive senescence. Biochim Biophys Acta 2010;1800:1121-1126.

47 Morris AA: Cerebral ketone body metabolism. J Inherit Metab Dis 2005;28:109-121.

48 Hsu TT, Leiske DL, Rosenfeld L, Sonner JM, Fuller GG: 3-Hydroxybutyric Acid Interacts with Lipid Monolayers at Concentrations That Impair Consciousness. Langmuir 2013;29:1948-1955.

49 Yang L, Zhao J, Milutinovic PS, Brosnan RJ, Eger EI, 2nd, Sonner JM: Anesthetic properties of the ketone bodies beta-hydroxybutyric acid and acetone. Anesth Analg 2007;105:673-679.

50 Billard JM: D-Serine in the aging hippocampus. J Pharm Biomed Anal 2015;116:18-24. 